\title{
Acknowledgement of manuscript reviewers 2015
}

Maged N Kamel Boulos ${ }^{*}$

The editor of International Journal of Health Geographics would like to thank all our reviewers who have contributed to the journal in Volume 14 (2015).

\section{Geir Aamodt \\ Norway}

Philip Abdelmalik

Canada

Charles N Agoti

Kenya

Jared Aldstadt

USA

Neal Alexander

Colombia

Luk Arbuckle

Canada

\section{Martijn Arns}

Netherlands

Vishal Arora

UK

Ananda Sankar Bandyopadhyay

USA

David Berrigan

USA

Janne Boone-Heinonen

USA

Manfred Buchroithner

Germany

Anibal Carbajo

Argentina

\section{Marilia Carvalho}

Brazil

Steve Carver

UK

Neil Coffee

Australia

Michael L Cohen

USA

\section{Natalie Colabianchi \\ USA}

Fabrice Courtin

France

Diego F Cuadros

Qatar

\section{Andrew Curtis}

USA

Paul Delamater

USA

\section{Kenneth Denike \\ Canada}

Naomi Lince-Deroche

South Africa

James Dunn

Canada

Lutz Ehlkes

Germany

\author{
Khaled El Emam \\ Canada
}

Jean-Philippe Empana

France

Chellafe Ensoy

Belgium

Roger Evans

UK

\begin{abstract}
Abby Flynt
USA
\end{abstract}

Joseph Gibbons

USA

Jianya Gong

China

William B Grant

USA

George Grekousis

Greece

\section{Aline Guttmann \\ France}

Michael Hagenlocher

Germany

Kobus Herbst

South Africa

Christian Herrmann

Switzerland

*Correspondence: maged.kamelboulos@uhi.ac.uk

University of the Highlands and Islands, Inverness, UK 


\begin{tabular}{|c|c|c|}
\hline Gary Higgs & Ann Klassen & Liang Mao \\
\hline UK & USA & USA \\
\hline $\begin{array}{l}\text { James Holt } \\
\text { USA }\end{array}$ & $\begin{array}{l}\text { Christian Klaus } \\
\text { USA }\end{array}$ & $\begin{array}{l}\text { Inocencio Daniel Maramba } \\
\text { Uk }\end{array}$ \\
\hline $\begin{array}{l}\text { Douglas Houston } \\
\text { USA }\end{array}$ & $\begin{array}{l}\text { Tom Koch } \\
\text { Canada }\end{array}$ & $\begin{array}{l}\text { Mariel Asbury Marlow } \\
\text { USA }\end{array}$ \\
\hline $\begin{array}{l}\text { Rebekah Huber } \\
\text { USA }\end{array}$ & $\begin{array}{l}\text { Michelle Condo } \\
\text { USA }\end{array}$ & $\begin{array}{l}\text { Tony Mathys } \\
\text { UK }\end{array}$ \\
\hline $\begin{array}{l}\text { Andrew Hudson-Smith } \\
\text { UK }\end{array}$ & $\begin{array}{l}\text { Ourania Kounadi } \\
\text { Austria }\end{array}$ & $\begin{array}{l}\text { Soumya Mazumdar } \\
\text { Australia }\end{array}$ \\
\hline $\begin{array}{l}\text { Alvaro Iruin } \\
\text { Spain }\end{array}$ & $\begin{array}{l}\text { Petr Kubicek } \\
\text { Czech Republic }\end{array}$ & $\begin{array}{l}\text { Paul Mccrorie } \\
\text { UK }\end{array}$ \\
\hline $\begin{array}{l}\text { Enrico Ivaldi } \\
\text { Italy }\end{array}$ & $\begin{array}{l}\text { Martin Kulldorff } \\
\text { USA }\end{array}$ & $\begin{array}{l}\text { Noreen Mcdonald } \\
\text { USA }\end{array}$ \\
\hline $\begin{array}{l}\text { Marta M Jankowska } \\
\text { USA }\end{array}$ & $\begin{array}{l}\text { Mei-Po Kwan } \\
\text { USA }\end{array}$ & $\begin{array}{l}\text { Moran Mika } \\
\text { Israel }\end{array}$ \\
\hline $\begin{array}{l}\text { Caroline Jeffery } \\
\text { UK }\end{array}$ & $\begin{array}{l}\text { Joseph Larmarange } \\
\text { France }\end{array}$ & $\begin{array}{l}\text { Michelle Morris } \\
\text { UK }\end{array}$ \\
\hline $\begin{array}{l}\text { Jeremie Jegu } \\
\text { UK }\end{array}$ & $\begin{array}{l}\text { Ryan Lash } \\
\text { USA }\end{array}$ & $\begin{array}{l}\text { Sarah-Anne Munoz } \\
\text { UK }\end{array}$ \\
\hline $\begin{array}{l}\text { Seol Young Jeong } \\
\text { Republic of Korea }\end{array}$ & $\begin{array}{l}\text { Veerle Lejon } \\
\text { France }\end{array}$ & $\begin{array}{l}\text { Bronwyn Myers } \\
\text { Australia }\end{array}$ \\
\hline $\begin{array}{l}\text { Yue Jin } \\
\text { USA }\end{array}$ & $\begin{array}{l}\text { Liliana Leone } \\
\text { Italy }\end{array}$ & $\begin{array}{l}\text { Keiko Nakamura } \\
\text { Japan }\end{array}$ \\
\hline $\begin{array}{l}\text { Andy Jones } \\
\text { UK }\end{array}$ & $\begin{array}{l}\text { Daniel Lewis } \\
\text { UK }\end{array}$ & $\begin{array}{l}\text { Robin Nesbitt } \\
\text { Germany }\end{array}$ \\
\hline $\begin{array}{l}\text { Rena Jones } \\
\text { USA }\end{array}$ & $\begin{array}{l}\text { Yan Lin } \\
\text { USA }\end{array}$ & $\begin{array}{l}\text { Dennis Nicholas } \\
\text { USA }\end{array}$ \\
\hline $\begin{array}{l}\text { Inkyung Jung } \\
\text { Republic of Korea }\end{array}$ & $\begin{array}{l}\text { Robert Lipton } \\
\text { USA }\end{array}$ & $\begin{array}{l}\text { Oliver O'Brien } \\
\text { UK }\end{array}$ \\
\hline $\begin{array}{l}\text { Lucie Kalousova } \\
\text { USA }\end{array}$ & $\begin{array}{l}\text { Jonathan London } \\
\text { USA }\end{array}$ & $\begin{array}{l}\text { Lisel O'Dwyer } \\
\text { Australia }\end{array}$ \\
\hline $\begin{array}{l}\text { Maged N Kamel Boulos } \\
\text { UK }\end{array}$ & $\begin{array}{l}\text { Valerie R Louis } \\
\text { Germany }\end{array}$ & $\begin{array}{l}\text { Georgiana Onicescu } \\
\text { USA }\end{array}$ \\
\hline $\begin{array}{l}\text { Steve Kammerer } \\
\text { USA }\end{array}$ & $\begin{array}{l}\text { Hui Luan } \\
\text { Canada }\end{array}$ & $\begin{array}{l}\text { Sophie Orton } \\
\text { UK }\end{array}$ \\
\hline $\begin{array}{l}\text { Nestor Kapusta } \\
\text { Austria }\end{array}$ & $\begin{array}{l}\text { Sandra MacRury } \\
\text { UK }\end{array}$ & $\begin{array}{l}\text { Ann Pascoe } \\
\text { UK }\end{array}$ \\
\hline $\begin{array}{l}\text { Hassan Karimi } \\
\text { USA }\end{array}$ & $\begin{array}{l}\text { Ravi Maheswaran } \\
\text { UK }\end{array}$ & $\begin{array}{l}\text { Lucy A Peipins } \\
\text { USA }\end{array}$ \\
\hline $\begin{array}{l}\text { P Kasemsuppakorn } \\
\text { Thailand }\end{array}$ & $\begin{array}{l}\text { Alessandro Mannelli } \\
\text { Italy }\end{array}$ & $\begin{array}{l}\text { Ronit Peled } \\
\text { Israel }\end{array}$ \\
\hline
\end{tabular}




Gavin Pereira
Australia
Antoni Pérez-Navarro
Spain
Revati Phalkey
Germany
Hugo Pilkington
France
Bram Piot
Senegal
Andrea Presotto
USA
Matthew Quick
Canada
Gita Ramjee
South Africa
Bernd Resch
Germany
Jennifer Roberts
USA
Juan D Rodas
Colombia
Francisco Miranda Rodrigues
Portugal
Mark Rosenberg
Canada
Corrine Ruktanonchai
UK
Undrew Rundle

\begin{tabular}{|c|}
\hline $\begin{array}{l}\text { Gerard Rushton } \\
\text { USA }\end{array}$ \\
\hline $\begin{array}{l}\text { Paul Salze } \\
\text { France }\end{array}$ \\
\hline $\begin{array}{l}\text { Guido Sanguinetti } \\
\text { UK }\end{array}$ \\
\hline $\begin{array}{l}\text { Nadine Schuurman } \\
\text { Canada }\end{array}$ \\
\hline $\begin{array}{l}\text { Vincent Seaman } \\
\text { USA }\end{array}$ \\
\hline $\begin{array}{l}\text { Safraj Shahul Hameed } \\
\text { India }\end{array}$ \\
\hline $\begin{array}{l}\text { Wei Shi } \\
\text { Canada }\end{array}$ \\
\hline $\begin{array}{l}\text { Timo Stübig } \\
\text { Germany }\end{array}$ \\
\hline $\begin{array}{l}\text { Wayne Sullender } \\
\text { USA }\end{array}$ \\
\hline $\begin{array}{l}\text { Hongbing Tao } \\
\text { China }\end{array}$ \\
\hline $\begin{array}{l}\text { Samuel D Towne Jr } \\
\text { USA }\end{array}$ \\
\hline $\begin{array}{l}\text { Gisela Van Kessel } \\
\text { Australia }\end{array}$ \\
\hline $\begin{array}{l}\text { Jean-Francois Viel } \\
\text { France }\end{array}$ \\
\hline $\begin{array}{l}\text { Ari Voutilainen } \\
\text { Finland }\end{array}$ \\
\hline $\begin{array}{l}\text { John Vulule } \\
\text { Kenya }\end{array}$ \\
\hline
\end{tabular}

Edward Walker

USA

Neng Wan

USA

Fahui Wang

USA

Tai-Chi Wang

Taiwan

Hannah Weir

USA

Laura White

USA

Michael Widener

Canada

Faustine Williams

USA

Michael Wimberly

USA

Meghan Winters

Canada

Chuanhua Yu

China

Ching-Syang Yue

Taiwan

Xingyou Zhang

USA

Li Zhu

USA

Marsil Zook

Australia 